\title{
Dispersion Lemmas Revisited
}

\author{
I. GASSER ${ }^{\mathrm{a}, *}$, P. A. MARKOWICH ${ }^{\mathrm{b}}$ and B. PERTHAME ${ }^{\mathrm{c}}$ \\ ${ }^{a}$ Universität Hamburg, Institut f. Angew. Mathematik, Bundesstraße 55, 20146 Hamburg, Germany; \\ ${ }^{\mathrm{b}}$ Johannes Kepler Universität Linz, Institut für Analysis und Numerik, Altenberger Straße 69, 4040 Linz, Austria; \\ ${ }^{\mathrm{c}}$ Ecole Normale Superieure, DMI, 45, rue d'Ulm, 75230 Paris Cedex 05, France
}

(Received 13 August 1997; In final form 1 December 1998)

\begin{abstract}
We investigate regularizing dispersive effects for various classical equations, e.g., the Schrödinger and Dirac equations. After Wigner transform, these dispersive estimates are reduced to moment lemmas for kinetic equations. They yield new regularization results for the Schrödinger equation (valid up to the semiclassical limit) and the Dirac equation.
\end{abstract}

Keywords: Dispersion lemmas, Schrödinger equation, Wigner equation, quantum-hydrodynamics, Dirac equation

\section{INTRODUCTION}

In this paper we investigate dispersive effects for various classical equations (Schrödinger, Dirac equations). We reduce these equations, after a Wigner transform, to a kinetic type equation

$$
\partial_{t} f+a(x, \xi) *_{x} \nabla_{x} f=g .
$$

Then, we apply to (1.1) the same multiplier method introduced in [13] in the context of pure Schrödinger and kinetic equations. We show that it yields various new results. For the Schrödinger equation with potential, we obtain regularizing effects in $L_{t}^{2}\left(H_{x, \text { loc }}^{1 / 2}\right)$ valid up to the semiclassical limit for potentials $V$ satisfying some kind of local one-sided
Lipschitz condition. These include Morawetz type estimates and seem to be optimal in the semiclassical limit. They are independent and complete those of Constantin and Saut [5] for merely bounded potentials, or those of Ruiz and Vega [18]; Sjögren and Sjölin [21]; Ben-Arzi and Klainerman [1].

The case of the Dirac equation also yields new results. They are obtained by reducing the Dirac equation, written in the form of a hyperbolic system, to a pseudo-differential equation $i u_{t}=P(|D|) u$ with a real and radial symbol $P(|\xi|)$. Then, we show that the dispersive effects for this equation can be proved through the classical Wigner transform and with a modification of the usual multiplier $(x /|x|) \cdot \xi$. This result extends those of Colin [3] and also provides a more standard approach.

${ }^{*}$ Corresponding author. 
We also would like to point out that our results combine and extend several ideas introduced in Gérard, Mauser, Markowich and Poupaud [9], concerning the approach to the Schrödinger and Dirac equations through Wigner transform, and in Lions and Perthame [13] concerning the analogy between dispersive and moment estimates. This kind of dispersive estimates where first established by Sjölin [20]; Constantin and Saut [4].

\section{DISPERSION LEMMAS FOR SCHRÖDINGER-TYPE PROBLEMS}

The subsequent results will be based on the theory of the Wigner-transform, for which we state here only the most basic (and important) properties. For an in-depth analysis we refer to $[9,12]$.

Let $f \in S^{\prime}\left(\mathbb{R}^{n} ; \mathbb{C}\right)$. Then the Wigner transform of $f$ on the scale $\varepsilon>0$ is given by

$$
\begin{aligned}
& w^{\varepsilon}[f](x, \xi) \\
& \quad=\frac{1}{(2 \pi)^{n}} \int_{\mathbb{R}^{n}} \bar{f}\left(x+\frac{\varepsilon}{2} v\right) f\left(x-\frac{\varepsilon}{2} v\right) \mathrm{e}^{i v \cdot \varepsilon} d v
\end{aligned}
$$

("-" denotes complex conjugation).

Note that $w^{\varepsilon}[f] \in S^{\prime}\left(\mathbb{R}_{x}^{n} \times \mathbb{R}_{\xi}^{n} ; \mathbb{R}\right)$. It is well known that, in general, $w^{\varepsilon}[f]$ is not a positive measure.

For the following we use the definition

$$
\hat{f}(\xi):=\left(\mathcal{F}_{x \rightarrow \xi} f\right)(\xi)=\int_{\mathbb{R}^{n}} f(x) \mathrm{e}^{-i x \cdot \xi} d x
$$

for the Fourier-transform on $\mathbb{R}^{n}$. Its inverse is denoted by

$$
\hat{g}(x):=\left(\mathcal{F}_{\xi \rightarrow x}^{-1} g\right)(x)=\frac{1}{(2 \pi)^{n}} \int_{\mathbb{R}^{n}} g(\xi) \mathrm{e}^{i x \cdot \xi} d \xi .
$$

Trivially, we have

$$
\left(\mathcal{F}_{\xi \rightarrow v} w^{\varepsilon}[f]\right)(x, v)=\bar{f}\left(x+\frac{\varepsilon}{2} v\right) f\left(x-\frac{\varepsilon}{2} v\right)
$$

and, after a simple computation

$$
\begin{aligned}
& \left(\mathcal{F}_{x \rightarrow \zeta} \boldsymbol{w}^{\varepsilon}[f]\right)(\zeta, \xi) \\
& \quad=\frac{1}{(2 \pi \varepsilon)^{m}} \hat{f}\left(\frac{\xi}{\varepsilon}+\frac{\zeta}{2}\right) \overline{\hat{f}}\left(\frac{\xi}{\varepsilon}-\frac{\zeta}{2}\right) .
\end{aligned}
$$

We conclude

$$
\begin{aligned}
& \int_{\mathbb{R}^{n}} w^{\varepsilon}[f] d \xi=|f(x)|^{2} \\
& \int_{\mathbb{R}^{n}} w^{\varepsilon}[f] d x=\frac{1}{(2 \pi \varepsilon)^{m}}\left|\hat{f}\left(\frac{\xi}{\varepsilon}\right)\right|^{2} .
\end{aligned}
$$

At first we consider the Schrödinger equation on $\mathbb{R}^{n}$ with a given real valued potential $V(x)$ :

$$
\begin{gathered}
i \varepsilon u_{t}=-\frac{\varepsilon^{2}}{2} \Delta u+V(x) u, \quad x \in \mathbb{R}^{n}, \quad t \in \mathbb{R}, \\
u(t=0)=u_{I} \quad \text { on } \mathbb{R}^{n} .
\end{gathered}
$$

It is verified by a simple calculation that $w(x, \xi, t):=$ $w^{\varepsilon}\left[u^{\varepsilon}(t)\right](x, \xi)$ satisfies the transport equation

$$
w_{t}+\xi \cdot \nabla_{x} w+\Theta[V] w=0, \quad(x, \xi) \in \mathbb{R}^{2 n}, \quad t \in \mathbb{R}
$$

$$
w(t=0)=w^{\varepsilon}\left[u_{I}\right] \text { on } \mathbb{R}^{2 n},
$$

where $\Theta[V]$ is given by

$$
\begin{aligned}
& (\Theta[V] f)(x, \xi) \\
& =-\frac{i}{(2 \pi)^{n}} \int_{\mathbb{R}^{n}} \frac{V\left(x+\frac{\varepsilon}{2} v\right)-V\left(x-\frac{\varepsilon}{2} v\right)}{\varepsilon} \\
& \bar{f}\left(x+\frac{\varepsilon}{2} v\right) f\left(x-\frac{\varepsilon}{2} v\right) \mathrm{e}^{i v \xi} d v .
\end{aligned}
$$

We shall now derive dispersive inequalities for the Schrödinger equation (2.2) by employing a multiplier technique on the Wigner equation (2.3). Although the subsequent results can also be obtained by using a multiplier technique directly 
on the Schrödinger equation, the 'detour' via the Wigner equation has two advantages. Firstly, it uses the connection between moment lemmas for kinetic equations and dispersion lemmas for Schrödinger type equations employing a somewhat intuitive multiplier on the kinetic level. Secondly it serves as a guideline for pseudo differential equations with more general symbols where a direct multiplier approach without " $k i$ netic detour" would even be less intuitive than in the Schrödinger case.

The first dispersion statement reads

TheOrem 2.1 For $n \geq 2$ let $\left(-\varepsilon^{2} / 2\right) \Delta+V$ be essentially self-adjoint and $V(x) \geq$ a.e. in $\mathbb{R}^{n}, V \in$ $W_{\text {loc }}^{1,1}\left(\mathbb{R}^{n} ; \mathbb{R}\right)$. Also assume $u_{I} \in D\left(\left(-\frac{\varepsilon^{2}}{2} \Delta+V\right)^{1 / 4}\right)$ $\subseteq L^{2}\left(\mathbb{R}^{n}\right) \quad$ and $\quad\left(\left[\left(x-x_{0}\right) \cdot \nabla V(x)\right]^{+} /\left|x-x_{0}\right|\right) /$ $(1+\sqrt{V(x)}) \in L^{\infty}\left(\mathbb{R}^{n}\right)$ for some $x_{0} \in \mathbb{R}^{n}$. Then there are constants $C_{n}$ independent of $x_{0}$ and $\varepsilon$ such that for all $-\infty<\tau_{1}<\tau_{2}<\infty$ we have

$$
\begin{aligned}
& \int_{\tau_{1}}^{\tau_{2}} \int_{\mathbb{R}^{2}} {\left[\varepsilon^{2}\left(\frac{|\nabla u|^{2}}{\left|x-x_{0}\right|}-\frac{\left|\left(x-x_{0}\right) \cdot \nabla u\right|^{2}}{\left|x-x_{0}\right|^{3}}\right)\right.} \\
&\left.-\left(\frac{x-x_{0}}{\left|x-x_{0}\right|} \cdot \nabla V(x)\right)|u|^{2}\right] d x d t \\
& \leq\left.C_{2}||\left(-\frac{\varepsilon^{2}}{2} \Delta+V\right)^{\frac{1}{4}} u_{I}\right|_{L^{2}\left(\mathbb{R}^{2}\right)} ^{2}, \quad(n=2) \\
& \int_{\tau_{1}}^{\tau_{2}}\left[\int_{\mathbb{R}^{3}} \varepsilon^{2}\left(\frac{|\nabla u|^{2}}{\left|x-x_{0}\right|}-\frac{\left|\left(x-x_{0}\right) \cdot \nabla u\right|^{2}}{\left|x-x_{0}\right|^{3}}\right) d x\right. \\
&+4 \pi \varepsilon^{2}\left|u\left(x_{0}, t\right)\right|^{2} \\
&\left.-\int_{\mathbb{R}^{3}}\left(\frac{x-x_{0}}{\left|x-x_{0}\right|} \cdot \nabla V(x)\right)|u|^{2} d x\right] d t \\
& \leq C_{3} \mid\left(-\frac{\varepsilon^{2}}{2} \Delta+V\right)^{\frac{1}{4}} u_{I} \|_{L^{2}\left(\mathbb{R}^{3}\right)}^{2}, \quad(n=3) \\
& \int_{\tau_{1}}^{\tau_{2}} \int_{\mathbb{R}^{n}}\left[\varepsilon ^ { 2 } \left(\frac{|\nabla u|^{2}}{\left|x-x_{0}\right|}\right.\right. \\
&\left.-\frac{\left|\left(x-x_{0}\right) \cdot \nabla u\right|^{2}}{\left|x-x_{0}\right|^{3}}+\frac{|u|^{2}}{\left|x-x_{0}\right|^{3}} \alpha_{n}\right)
\end{aligned}
$$

$$
\begin{aligned}
& \left.-\left(\frac{x-x_{0}}{\left|x-x_{0}\right|} \cdot \nabla V(x)\right)|u|^{2}\right] d x d t \\
\leq & C_{n}\left\|\left(-\frac{\varepsilon^{2}}{2} \Delta+V\right)^{\frac{1}{4}} u_{I}\right\|_{L^{2}\left(\mathbb{R}^{n}\right)}^{2}, \quad(n \geq 4) \\
\alpha_{n}= & \frac{n^{2}-4 n+3}{4} .
\end{aligned}
$$

Here and in the sequel we denote by $f^{+}, f^{-}$the positive and the negative part of $f$, i.e., $f=f^{+}-f^{-}$.

Proof We multiply the transport equation (2.3a) by $\left(x-x_{0} /\left|x-x_{0}\right|\right) \cdot \xi$ and integrate over $\mathbb{R}_{\xi}^{n} \times$ $\mathbb{R}_{x}^{n} \times\left(\tau_{1}, \tau_{2}\right)$. We obtain

$$
\begin{aligned}
& \int_{\tau_{1}}^{\tau_{2}} \int_{\mathbb{R}^{2 n}} \xi \cdot \nabla_{x}\left(\frac{\left(x-x_{0}\right) \cdot \xi}{\left|x-x_{0}\right|}\right) w d \xi d x d t \\
& -\int_{\tau_{1}}^{\tau_{2}} \int_{\mathbb{R}^{2 n}} \frac{x-x_{0}}{\left|x-x_{0}\right|} \cdot \xi \Theta[V] w d \xi d x d t \\
& \quad=\int_{\mathbb{R}^{n}} \frac{x-x_{0}}{\left|x-x_{0}\right|} \cdot\left(J\left(x, \tau_{2}\right)-J\left(x, \tau_{1}\right)\right) d x
\end{aligned}
$$

where we used

$$
|u(x, t)|^{2}=\int_{\mathbb{R}^{n}} w(x, \xi, t) d \xi
$$

and set

$$
J(x, t):=\int_{\mathbb{R}^{n}} \xi w(x, \xi, t) d \xi .
$$

The first term on the left hand side of (2.6) was already computed in the reference [13] in terms of the wave function $u$. It is given by those terms on the left hand side of (2.5), which do not involve $\nabla V(x)$. Using (2.4) we obtain

$$
\int_{\mathbb{R}^{n}} \xi \Theta[V] f d \xi=\nabla_{x} V(x)|f(x)|^{2}
$$

which implies

$$
\begin{aligned}
& \int_{\tau_{1}}^{\tau_{2}} \int_{\mathbb{R}^{2 n}} \frac{x-x_{0}}{\left|x-x_{0}\right|} \cdot \xi \Theta[V] w d \xi d x d t \\
& \quad=\int_{\tau_{1}}^{\tau_{2}} \int_{\mathbb{R}^{n}} \frac{x-x_{0}}{\left|x-x_{0}\right|} \cdot \nabla V(x)|u(x, t)|^{2} d x d t .
\end{aligned}
$$


Another simple calculation (using the definition of the Wigner-transform) gives

$$
J(x, t)=\varepsilon \operatorname{Im}(\bar{u}(x, t) \nabla u(x, t))
$$

so that

$$
\begin{aligned}
& \int_{\mathbb{R}^{n}} \frac{x-x_{0}}{\left|x-x_{0}\right|} \cdot J(x, T) d x \\
& \quad=\varepsilon \operatorname{Im} \int_{\mathbb{R}^{n}}\left(\bar{u}(x, T) \frac{x-x_{0}}{\left|x-x_{0}\right|}\right) \cdot \nabla u(x, T) d x
\end{aligned}
$$

and

$$
\begin{aligned}
& \left|\int_{\mathbb{R}^{n}} \frac{x-x_{0}}{\left|x-x_{0}\right|} \cdot J(x, T) d x\right| \\
& \quad \leq \varepsilon|\nabla u(T)|_{-(1 / 2)}\left|\frac{x-x_{0}}{\left|x-x_{0}\right|} u(T)\right|_{(1 / 2)} .
\end{aligned}
$$

Here we denote the (semi) norms on $H^{\alpha}\left(\mathbb{R}^{n}\right)$ by

$$
\begin{aligned}
|f|_{\alpha}^{2} & :=\int_{\mathbb{R}^{n}}|\hat{f}(\xi)|^{2}|\xi|^{2 \alpha} d \xi \\
\|f\|_{\alpha}^{2} & =\int_{\mathbb{R}^{n}}\left(1+|\xi|^{2}\right)^{\alpha}|\hat{f}(\xi)|^{2} d \xi
\end{aligned}
$$

for $\alpha \in \mathbb{R}$. Then we deduce from the above inequality

$$
\begin{aligned}
& \left|\int_{\mathbb{R}^{n}} \frac{x-x_{0}}{\left|x-x_{0}\right|} \cdot J(x, T) d x\right| \\
& \quad \leq \varepsilon|u(T)|_{1 / 2}\left|\frac{x-x_{0}}{\left|x-x_{0}\right|} u(T)\right|_{1 / 2} .
\end{aligned}
$$

Now we consider the operators

$$
R_{l}: f \rightarrow \frac{x_{l}}{|x|} f, \quad l=1, \ldots, n .
$$

We compute

$$
\left|R_{l} f\right|_{1 / 2}^{2}=a_{n}^{2} \int_{\mathbb{R}^{n}}\left|\lim _{\delta \rightarrow 0} \int_{|\omega|>\delta} \frac{\omega_{l}}{|\omega|^{n+1}} \hat{f}(\xi-\omega) d \omega\right|^{2}|\xi| d \xi .
$$

$a_{n}$ only depends on the dimension $n$. Here we used $|\widehat{x}|(\xi)=a_{n}\left(|\xi|^{-1-n}\right)^{\bullet}$ where "•" denotes the exten- sion to $\xi=0$. Since

$$
g \rightarrow \lim _{\delta \rightarrow 0} \int_{|\omega|>\delta} \frac{\omega_{l}}{|\omega|^{n+1}} g(\xi-\omega) d \omega
$$

is the Riesz-transformation (with index $l$ ) up to a multiplicative constant, it is bounded on $L^{2}\left(\mathbb{R}^{n}\right)(c f$. [S, II, 4.2, Theorem 3]). Thus, it is also bounded on $L^{2}\left(\mathbb{R}^{n},|\xi|^{\alpha} d \xi\right)$, where $-n<\alpha<n(c f$. [S, II, 6.3]) and

$$
\left|R_{l} f\right|_{1 / 2} \leq C|f|_{1 / 2}
$$

follows. We obtain

$$
\left|\int_{\mathbb{R}^{n}} \frac{x-x_{0}}{\left|x-x_{0}\right|} \cdot J(x, T) d x\right| \leq C \varepsilon|u(T)|_{1 / 2}^{2}
$$

with a constant $C$, which only depends on $n$. It remains to show the transport of $\sqrt{\varepsilon} u|(t)|_{(1 / 2)}$ by the Schrödinger flow with an $\varepsilon$-independent constant. We denote by $S(t)$ the solution operator of (2.2), i.e., $S(t) u_{I}$ is the solution $u(t)$ of (2.2) at time $t$. We have

$$
\|S(t)\|_{L^{2}\left(\mathbb{R}^{n}\right) \rightarrow L^{2}\left(\mathbb{R}^{n}\right)}=1 \quad \forall t \in \mathbb{R}
$$

and we conclude

$$
\begin{aligned}
& \left\|\left(-\frac{\varepsilon^{2}}{2} \Delta+V\right)^{1 / 4} S(t) u_{I}\right\|_{L^{2}\left(\mathbb{R}^{n}\right)} \\
& =\left\|\left(-\frac{\varepsilon^{2}}{2} \Delta+V\right)^{1 / 4} u_{I}\right\|_{L^{2}\left(\mathbb{R}^{n}\right)} \quad \forall t \in \mathbb{R} .
\end{aligned}
$$

Since $V \geq 0$ we have

$$
\left\|\left(-\frac{\varepsilon^{2}}{2} \Delta\right)^{1 / 4} \varphi\right\|_{L^{2}\left(\mathbb{R}^{n}\right)} \leq\left\|\left(-\frac{\varepsilon^{2}}{2} \Delta+V\right)^{1 / 4} \varphi\right\|_{L^{2}\left(\mathbb{R}^{n}\right)}
$$

for all $\varphi \in D\left(\left(-\left(\varepsilon^{2} / 2\right) \Delta+V\right)^{(1 / 4)}\right)$ (see [17], p. 44, prop. 9). Setting $\varphi=u(t)$ gives

$$
\begin{aligned}
& \sqrt{\varepsilon}|u(t)|_{1 / 2} \leq 2^{1 / 4}\left\|\left(-\frac{\varepsilon^{2}}{2} \Delta+V\right)^{1 / 4} u_{I}\right\|_{L^{2}\left(\mathbb{R}^{n}\right)} \\
& \forall t \in \mathbb{R}
\end{aligned}
$$


and by proceeding analogously

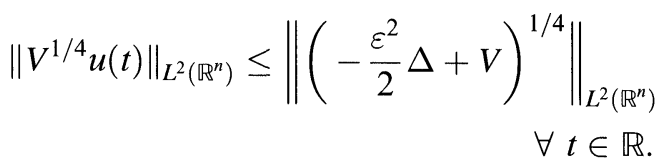

From (2.8) we derive:

$$
\begin{aligned}
& \left|\int_{\mathbb{R}^{n}} \frac{x-x_{0}}{\left|x-x_{0}\right|} \cdot J(x, T) d x\right| \\
& \quad \leq C\left\|\left(-\frac{\varepsilon^{2}}{2} \Delta+V\right)^{1 / 4} u_{I}\right\|_{L^{2}\left(\mathbb{R}^{n}\right)}^{2}
\end{aligned}
$$

and the assertion of Theorem (2.1) follows.

The next Theorem is concerned with the $x$ localized dispersion result for the Schrödinger equation (2.2) where the singularity at $x=x_{0}$ in (2.5) is removed.

Theorem 2.2 For $n \geq 1$ let the assumptions on $V$ and $u_{I}$ of Theorem (2.1) hold. Then for $0<\alpha \leq 1$ three is $D=D(n, \alpha)>0$ independent of $\varepsilon$ such that for all $-\infty<\tau_{1}<\tau_{2}<\infty$ :

$$
\begin{aligned}
& \varepsilon^{2} \int_{\tau_{1}}^{\tau_{2}} \int_{\mathbb{R}^{n}} \frac{|\nabla u|^{2}}{\left(1+\left|x-x_{0}\right|^{\alpha}\right)^{1+1 / \alpha}} d x d t \\
& -\int_{\tau_{1}}^{\tau^{2}} \int_{\mathbb{R}^{n}} \frac{\left(x-x_{0}\right) \cdot \nabla V(x)}{\left(1+\left|x-x_{0}\right|^{\alpha}\right)^{1 / \alpha}}|u|^{2} d x d t \\
& \quad \leq D\left\|\left(-\frac{\varepsilon^{2}}{2} \Delta+V\right)^{1 / 4} u_{I}\right\|_{L^{2}\left(\mathbb{R}^{n}\right)}^{2}
\end{aligned}
$$

Proof As in [13] we multiply (2.3a) by $(x \cdot \xi /$ $\left(1+|x|^{\alpha}\right)^{(1 / \alpha)}$ ) (we set $x_{0}=0$ to simplify the formulas) and obtain

$$
\begin{aligned}
& \int_{\tau_{1}}^{\tau_{2}} \int_{\mathbb{R}^{2 n}} \xi \cdot \nabla_{x}\left(\frac{x \cdot \xi}{\left(1+|x|^{\alpha}\right)^{1 / \alpha}}\right) w d x d \xi d t \\
& \quad-\int_{\tau_{1}}^{\tau_{2}} \int_{\mathbb{R}^{n}} \frac{x \cdot \nabla V(x)}{\left(1+|x|^{\alpha}\right)^{1 / \alpha}}|u|^{2} d x d t \\
& =\int_{\mathbb{R}^{n}} \frac{x}{\left(1+|x|^{\alpha}\right)^{1 / \alpha}} \cdot\left(J\left(x, \tau_{2}\right)-J\left(x, \tau_{1}\right)\right) d x .
\end{aligned}
$$

The first term on the left hand side can be restated in the wave-function $u$ after a long and unpleasant calculation. We obtain

$$
\begin{aligned}
& \int_{\tau_{1}}^{\tau_{2}} \int_{\mathbb{R}^{2 n}} \xi \cdot \nabla_{x}\left(\frac{x \cdot \xi}{\left(1+|x|^{\alpha}\right)^{1 / \alpha}}\right) w d x d \xi d t \\
& =\varepsilon^{2} \int_{\tau_{1}}^{\tau_{2}} \int\left(\frac{|\nabla u|^{2}}{\left(1+|x|^{\alpha}\right)^{1 / \alpha}}-\frac{(x \cdot \nabla u)^{2}|x|^{\alpha-2}}{\left(1+|x|^{\alpha}\right)^{1+1 / \alpha}}\right) d x d t \\
& \quad+\varepsilon^{2} \int_{\tau_{1}}^{\tau_{2}} \int F_{\alpha}(x)|u|^{2} d x d t
\end{aligned}
$$

where $F_{\alpha}(x) \geq 0$ for $0<\alpha \leq 1(c f$. [3]). Thus it has the lower bound

$$
c_{\alpha} \varepsilon^{2} \int_{\tau_{1}}^{\tau_{2}} \int \frac{|\nabla u|^{2}}{\left(1+|x|^{\alpha}\right)^{(1+1 / \alpha)}} d x d t .
$$

with $c_{\alpha}>0$. Since $\left(\partial / \partial x_{l}\right) \quad\left(x_{j} /\left(1+|x|^{\alpha}\right)^{(1 / \alpha)}\right) \in$ $L^{\infty}\left(\mathbb{R}^{n}\right)$, the treatment of the term which involves $J$ in (2.10) is much simpler than of the one in Theorem 2.1.

Remark 2.1 Note that the term involving the electric field $\nabla V$ in (3.5) can be estimated (due to the assumptions of Theorem 2.1) by

$$
\begin{aligned}
\int_{\tau_{1}}^{\tau_{2}} & \int_{\mathbb{R}^{n}} \frac{x-x_{0}}{\left|x-x_{0}\right|} \cdot \nabla V(x)|u|^{2} d x d t \\
\leq & \int_{\tau_{1}}^{\tau_{2}} \int_{\mathbb{R}^{n}} \frac{\left[\left(x-x_{0}\right) \cdot \nabla V(x)\right]^{-}}{\left|x-x_{0}\right|}|u|^{2} d x d t \\
& \quad+\int_{\tau_{1}}^{\tau_{2}} \int_{\mathbb{R}^{n}}(1+\sqrt{V(x)})|u|^{2} d x d t
\end{aligned}
$$

and by interpolation:

$$
\begin{aligned}
& \int_{\tau_{1}}^{\tau^{2}} \int_{\mathbb{R}^{n}}(1+\sqrt{V(x)})|u|^{2} d x d t \\
& \leq\left(\tau_{2}-\tau_{1}\right)\left(\left\|u_{I}\right\|_{L^{2}\left(\mathbb{R}^{n}\right)}^{2}+\left\|\left(-\frac{\varepsilon^{2}}{2} \Delta+V\right)^{1 / 4} u_{I}\right\|_{L^{2}\left(\mathbb{R}^{n}\right)}^{2}\right) .
\end{aligned}
$$


Thus

$$
\begin{aligned}
\int_{\tau_{1}}^{\tau_{2}}\left[\int_{\mathbb{R}^{n}}(\right. & \varepsilon^{2}\left(\frac{|\nabla u|^{2}}{\left|x-x_{0}\right|}-\frac{\left|\left(x-x_{0}\right) \cdot \nabla u\right|^{2}}{\left|x-x_{0}\right|^{3}}\right) d x \\
& \left.+\left\{\begin{array}{ll}
0, & n=2 \\
4 \pi\left|u\left(x_{0}, t\right)\right|^{2}, & n=3 \\
\alpha_{n} \int_{\mathbb{R}^{n}} \frac{|u|^{2}}{\left|x-x_{0}\right|^{3}} d x & n \geq 4
\end{array}\right\}\right) \\
& \left.+\int_{\mathbb{R}^{n}} \frac{\left[\left(x-x_{0}\right) \cdot \nabla V(x)\right]^{-}}{\left|x-x_{0}\right|}|u|^{2} d x\right] d t \\
\leq & C_{n}\left(\tau_{2}-\tau_{1}\right)\left\|u_{I}\right\|_{L^{2}\left(\mathbb{R}^{n}\right)}^{2}+C_{n}\left(1+\tau_{2}-\tau_{1}\right) \\
& \times\left\|\left(-\frac{\varepsilon^{2}}{2} \Delta+V\right)^{1 / 4} u_{I}\right\|_{L^{2}\left(\mathbb{R}^{n}\right)}^{2}
\end{aligned}
$$

follows. The corresponding term in (2.9) is treated analogously.

Remark 2.2 The Theorems 2.1 and 2.2 contain qualitatively different statements. Theorem 2.2 (which is more along the lines of the dispersion lemmas found in the literature $[4,5,20,22])$ states that initial data with $\left\|\left(-\varepsilon^{2} / 2 \Delta+V\right)^{(1 / 4)} u_{I}\right\|_{L^{2}\left(\mathbb{R}^{n}\right)}$ $<\infty$ yield $L_{\text {loc }}^{1}\left(\mathbb{R}_{t} ; H_{\text {loc }}^{1}\left(\mathbb{R}_{x}^{n}\right)\right)$-solutions $u=u(x, t)$ (under the stated assumptions on the potential). Theorem 2.1 (which holds for dimensions $\geq 2$ ) instead has two main features. Firstly, the $x$ locality of Theorem 2.2 is replaced by a regularization property "away from the direction $\nabla u(x)=x-x_{0}$ " and secondly, there is the singularity of the left-hand side of (2.5) at $x=x_{0}(c f$. [3]).

Remark 2.3 A main point of both Theorems is the precise (i.e., optimal) dependence of the estimates on the scaled Planck-constant $\varepsilon$. Even for potentials $V \in L^{\infty}\left(\mathbb{R}^{n}\right)$ the approach of bootstrapping the dispersion results for the free Schrödinger equation by applying Duhamel's formula ( $c f$. [5]) gives results which are worse as far as the dependence of $\varepsilon$ is concerned.

Remark 2.4 The class of admissible potentials in the Theorems 2.1 and 2.2 is different from what was considered in the literature $[1,5,18,21]$. Note that, for example, the harmonic oscillator with potential $V(x)=|x|^{2}$ is admissible, while important shortrange potentials are excluded by the assumption
$V^{-} \in L^{\infty}$. This can somewhat be remedied at the expense of the optimality of the appearing constants in dependence of $\varepsilon$ since the positivity of the potential was only used to have a positive Hamiltonian. For example, we can easily prove

COROllary 2.1 Assume that multiplication by $|V(x)|$ is infinitesimally form bounded with respect to $-\Delta$ and that $\left([x \cdot \nabla V(x)]^{+} /\left(1+|x|^{\alpha}\right)^{(1 / \alpha)}\right) /$ $(1+\sqrt{|V(x)|}) \in L^{\infty}\left(\mathbb{R}^{n}\right)$ for some $0<\alpha \leq 1$. Also let $u_{I} \in H^{(1 / 2)}\left(\mathbb{R}^{n}\right)$ and $-\infty<\tau_{1}<\tau_{2}<\infty$. Then there is $E=E\left(n, \alpha, \tau_{1}, \tau_{2}, \varepsilon\right)>0$ such that

$$
\begin{aligned}
& \int_{\tau_{1}}^{\tau_{2}} \int_{\mathbb{R}^{n}} \frac{|\nabla u|^{2}}{1+|x|^{1+\alpha}} d x d t \\
& \quad+\int_{\tau_{1}}^{\tau_{2}} \int_{\mathbb{R}_{n}} \frac{[x \cdot \nabla V(x)]^{-}}{\left(1+|x|^{\alpha}\right)^{1 / \alpha}}|u|^{2} d x d t \leq E\left\|u_{I}\right\|_{1 / 2}^{2} .
\end{aligned}
$$

Also, certain time dependent potentials can easily be included in the theory.

Remark 2.5 (Nonlinear Schrödinger equation)

Consider the nonlinear IVP $(n \geq 2)$ :

$$
\begin{gathered}
i \varepsilon u_{t}=-\frac{\varepsilon^{2}}{2} \Delta u+h\left(|u|^{2}\right) u, \quad x \in \mathbb{R}^{n}, \quad t \in \mathbb{R} \\
u(t=0)=u_{I} \quad \text { in } \mathbb{R}^{n},
\end{gathered}
$$

with the real enthalpy function $h=h(\sigma), \sigma>0$. Using the multiplier $\left(x-x_{0}\right) /\left(\left|x-x_{0}\right|\right) \cdot \xi$ for the Wigner equation we obtain (after an integration by parts):

$$
\begin{aligned}
& \int_{\tau_{1}}^{\tau_{2}} \int_{\mathbb{R}^{2 n}} \xi \cdot \nabla\left(\frac{\left.\left(x-x_{0}\right) \cdot \xi\right)}{\left|x-x_{0}\right|}\right) w d x d \xi d t \\
& +(n-1) \int_{\tau_{1}}^{\tau_{2}} \int_{\mathbb{R}^{n}} \frac{p\left(|u|^{2}\right)}{\left|x-x_{0}\right|} d x d t \\
& \quad=\int_{\mathbb{R}^{n}} \frac{x-x_{0}}{\left|x-x_{0}\right|} \cdot\left(J\left(x, \tau_{2}\right)-J\left(x, \tau_{1}\right)\right) d x,
\end{aligned}
$$

where $p^{\prime}(\sigma)=h^{\prime}(\sigma) \sigma$. Also, the conserved total energy is

$$
E=\frac{\varepsilon^{2}}{2} \int_{\mathbb{R}^{n}}|\nabla u|^{2} d x+\int_{\mathbb{R}^{n}} H\left(|u|^{2}\right) d x,
$$


with $H^{\prime}=h$. Assume that $H(\sigma) \geq-K \sigma$ for some $K>0$ and $p(\sigma) \geq 0$. Then, since the first term on the left hand side of (2.13) is nonnegative and since the term on the right hand side is bounded by $2 E(t)+2\|u(t)\|_{L^{2}\left(\mathbb{R}^{n}\right)}^{2}$, we obtain

$$
\begin{aligned}
& \int_{-\infty}^{\infty} \int_{\mathbb{R}^{n}} \frac{p\left(|u(x, t)|^{2}\right)}{\left|x-x_{0}\right|} d x d t \\
& \leq \varepsilon^{2} \int_{\mathbb{R}^{n}}\left|\nabla u_{I}\right|^{2} d x+2 \int_{\mathbb{R}^{n}} H\left(\left|u_{I}\right|^{2}\right) d x \\
& \quad+2 \int_{\mathbb{R}^{n}}\left|u_{I}\right|^{2} d x .
\end{aligned}
$$

This is a classical estimate (of Morawetz-type), which can be found in $[6,14]$. Note that we also can bound the first term in (2.5) (involving the singularity at $x=x_{0}$ ) by a constant, which only depends on the dimension, the initial energy, and $\left\|u_{I}\right\|_{L^{2}\left(\mathbb{R}^{n}\right)}$.

Similar estimates [16] can also be obtained for the Schrödinger-Poisson problem $(c f .[12,15])$ and for Hartree-Fock equations [7].

Remark 2.6 (Quantum-hydrodynamics, $c f$. [8]) For some applications it is important to restate the estimates of Theorem 2.1 in terms of the macroscopic densities

$$
\rho(x, t)=|u(x, t)|^{2}
$$

and $J=J(x, t)$ defined in (2.7). We obtain after a lengthy calculation:

$$
\begin{aligned}
\int_{\tau_{1}}^{\tau_{2}}\left[\int _ { \mathbb { R } _ { x } ^ { n } } \left[\frac{1}{\rho\left|x-x_{0}\right|}\left(|J|^{2}-\frac{\left(\left(x-x_{0}\right) \cdot J\right)^{2}}{\left|x-x_{0}\right|^{2}}\right)\right.\right. \\
+\frac{\varepsilon^{2}}{4\left|x-x_{0}\right|}\left(|\nabla \rho|^{2}-\frac{\left(\left(x-x_{0}\right) \cdot \nabla \rho\right)^{2}}{\left|x-x_{0}\right|^{2}}\right) \\
\left.\quad-\frac{x-x_{0}}{\left|x-x_{0}\right|} \cdot \nabla V(x) \rho\right] d x \\
\left.+\left\{\begin{array}{cc}
8 \pi \varepsilon^{2} \rho\left(x_{0}, t\right), & n=3 \\
\alpha_{n} \varepsilon^{2} \int_{\mathbb{R}^{n}} \frac{\rho(x, t)}{\left|x-x_{0}\right|^{3}} d x, & n>3
\end{array}\right\}\right] d t \\
\leq C_{n}\left\|\left(-\frac{\varepsilon^{2}}{2} \Delta+V\right)^{1 / 4} u_{I}\right\|_{L^{2}\left(\mathbb{R}^{n}\right)}^{2} .
\end{aligned}
$$

Remark 2.7 (Schrödinger multiplier) Theorem 2.1 can also be proven by multiplying the Schrödinger equation by

$$
\frac{x-x_{0}}{\left|x-x_{0}\right|} \cdot \nabla \bar{u}+\frac{n-1}{2} \frac{1}{\left|x-x_{0}\right|} \bar{u},
$$

taking real parts and integrating by parts ( $c f$. [14]).

\section{RADIAL FOURIER MULTIPLIERS}

Finally we shall consider PDE's with a real radial Fourier multiplier $P$ on $\mathbb{R}^{n}$ :

$$
\begin{gathered}
i u_{t}=P(|D|) u, \quad x \in \mathbb{R}^{n}, \quad t \in \mathbb{R} \\
u(t=0)=u_{I} \quad \text { on } \mathbb{R}^{n} .
\end{gathered}
$$

The case $P(r)=r^{\nu}$ with $\nu \geq 2$ was analysed in [3] by using a specially constructed Wigner-type transform. Here we shall employ the standard Wigner transform (2.1) and allow a more general class of symbols ( $c f$. [2] for local smoothing results).

Theorem 3.1 Let $P \in C^{2}([0, \infty) ; \mathbb{R})$ and assume that the map

$$
\xi \rightarrow \sigma(\zeta, \xi):=\int_{-1 / 2}^{1 / 2} \frac{P^{\prime}(|\xi+s \zeta|)}{|\xi+s \zeta|}(\xi+s \zeta) d s
$$

is one-to-one and onto from $\mathbb{R}^{n}$ to $\mathbb{R}^{n}$ for all $\zeta \in \mathbb{R}^{n}$. Then the solution of (3.18) satisfies for all $x_{0} \in \mathbb{R}^{n}$

$$
\begin{gathered}
\int_{-\infty}^{\infty}\left[\int_{\mathbb{R}^{n}}\left(\frac{|\nabla u|^{2}}{\left|x-x_{0}\right|}-\frac{\left|\left(x-x_{0}\right) \cdot \nabla u\right|^{2}}{\left|x-x_{0}\right|^{3}}\right) d x\right. \\
\left.+\left\{\begin{array}{cr}
0, & n=2 \\
4 \pi\left|u\left(x_{0}, t\right)\right|^{2}, & n=3 \\
\alpha_{n} \int_{\mathbb{R}^{n}} \frac{|u|^{2}}{\left|x-x_{0}\right|^{3}} d x, & n \geq 4
\end{array}\right\}\right] d t \\
\left\{\begin{array}{ll}
\leq, & n=2 \\
=, & n \geq 3
\end{array}\right\} \frac{2}{(2 \pi)^{n}} \int_{\mathbb{R}^{n}} \frac{|\xi|^{2}}{\left|P^{\prime}(|\xi|)\right|}\left|\hat{u}_{I}(\xi)\right|^{2} d \xi,
\end{gathered}
$$


and for $0<\alpha \leq 1$

$$
\begin{aligned}
& \int_{-\infty}^{\infty} \int_{\mathbb{R}^{n}} \frac{|\nabla u|^{2}}{\left(1+|x|^{\alpha}\right)^{(1+1 / \alpha)}} d x d t \\
& \quad \leq C_{\alpha} \int_{\mathbb{R}^{n}} \frac{|\xi|^{2}}{\left|P^{\prime}(|\xi|)\right|}\left|\hat{u}_{I}(\xi)\right|^{2} d \xi \quad(n \geq 1) .
\end{aligned}
$$

Proof The evolution equation for the $x$-Fourier transform $\hat{w}(\zeta, \xi, t):=\mathcal{F}_{x \rightarrow \zeta} w^{1}[u(t)]$ (we set $\varepsilon=1$ in (2.1)) reads:

$$
\hat{w}_{t}+i \zeta \cdot \sigma(\zeta, \xi) \hat{w}=0, \quad \hat{w}(t=0)=\hat{w}_{I}
$$

(cf. [9]). We now define $\varphi=\varphi(r)$ by $\varphi^{\prime}(r)=(r /$ $\left.\left(1+r^{\alpha}\right)^{(1 / \alpha)}\right)$ or, alternatively, $\varphi(r)=r$ and compute

$$
\mathcal{F}_{x \rightarrow \zeta}\left(\xi \cdot \nabla_{x}\left(\xi \cdot \nabla_{\varphi}(|x|)\right)\right)=-(\xi \cdot \zeta)^{2} \varphi(\widehat{|x|})(\xi) .
$$

Next we test (3.3) against

$$
a(\zeta, \xi):=i \frac{(\xi \cdot \zeta)^{2}}{\zeta \cdot \sigma(\zeta, \xi)} \overline{\varphi(\widehat{|x|})}(\zeta)
$$

We obtain

$$
\begin{aligned}
& -\int_{\tau_{1}}^{\tau_{2}}\left\langle(\zeta \cdot \xi)^{2} \overline{\varphi(\widehat{|x|})}(\zeta), \hat{w}\right\rangle_{\xi, \zeta} d t \\
& =i\left\langle\frac{(\xi \cdot \zeta)^{2}}{\overline{\zeta \cdot \sigma(\zeta, \xi)}} \overline{\varphi(\widehat{|x|})}(\zeta), \hat{w}\left(\tau_{1}\right)\right\rangle_{\xi, \zeta} \\
& -i\left\langle\frac{(\xi \cdot \zeta)^{2}}{\zeta \cdot \sigma(\zeta, \xi)} \overline{\varphi(\widehat{|x|})}(\zeta), \hat{w}\left(\tau_{2}\right)\right\rangle_{\xi, \zeta} .
\end{aligned}
$$

With $\varphi(r)=r$ we have

$$
\begin{aligned}
& -\left\langle(\zeta \cdot \xi)^{2} \overline{\widehat{|x|}}(\zeta), \hat{w}\right\rangle_{\xi, \zeta} \\
& \quad=\int_{\mathbb{R}^{2 n}}\left(\frac{|\xi|^{2}}{|x|}-\frac{(\xi \cdot x)^{2}}{|x|^{3}}\right) w d \xi d x
\end{aligned}
$$

and with $\varphi^{\prime}(r)=r /\left(1+r^{\alpha}\right)^{(1 / \alpha)}$

$$
\begin{aligned}
-\left\langle(\zeta \cdot \xi)^{2} \overline{\varphi(\widehat{|x|})}(\zeta), \hat{w}\right\rangle_{\xi, \zeta} \\
=\int_{\mathbb{R}^{2 n}} \xi \cdot \nabla_{x}\left(\frac{x \cdot \xi}{\left(1+|x|^{\alpha}\right)^{1 / \alpha}}\right) w d \xi d x .
\end{aligned}
$$

Therefore, the work to express the left-hand side of (3.4) in terms of $u$ has already been done [13] and gives rise to the left hand sides of (3.2). For the computation of the right-hand side we note that (3.3) gives

$$
\hat{w}(\zeta, \xi, t)=\exp (-i \zeta \cdot \sigma(\zeta, \xi) t) \hat{w}_{I}(\zeta, \xi)
$$

We now carry out the change of variables from $\xi$ to $\sigma=\sigma(\zeta, \xi)$, denote $D=|\operatorname{det}(\partial \sigma / \partial \xi)|$ and obtain

$$
\begin{gathered}
i\left\langle\frac{(\xi \cdot \zeta)^{2}}{\zeta \cdot \sigma(\zeta, \xi)} \overline{\varphi(\widehat{|x|})}(\zeta), \hat{w}\left(\zeta, \xi, T_{l}\right)\right\rangle_{\xi, \zeta} \\
=i\left\langle\frac{(\xi(\zeta, \sigma) \cdot \zeta)^{2}}{\zeta \cdot \sigma} \overline{\varphi(\widehat{|x|})}(\zeta) D(\zeta, \sigma)^{-1}\right. \\
\left.\quad \exp \left(-i \zeta \cdot \sigma T_{l}\right), \hat{w}_{I}(\zeta, \xi(\zeta, \sigma))\right\rangle_{\sigma, \zeta}
\end{gathered}
$$

for $l=1,2$. We compute

$$
\begin{aligned}
& \lim _{T_{l} \rightarrow \pm \infty} i \zeta \overline{\varphi(\widehat{|x|})}(\zeta) \exp \left(-i \zeta \cdot \sigma T_{l}\right) \\
& =-\mathcal{F}_{x \rightarrow \zeta}\left(\lim _{T_{l} \rightarrow \pm \infty} \nabla \varphi\left(\mid x-\sigma T_{l}\right)\right) \\
& =-\mathcal{F}_{x \rightarrow \zeta}\left(\lim _{T_{l} \rightarrow \pm \infty} \operatorname{sgn}\left(T_{l}\right) \frac{\sigma}{|\sigma|}\right) \\
& =\frac{\sigma}{|\sigma|} \delta(\zeta)\left\{\begin{array}{ll}
+1, & T_{l} \rightarrow-\infty \\
-1, & T_{l} \rightarrow+\infty
\end{array}\right\} .
\end{aligned}
$$

Also we obtain

$$
\begin{aligned}
& L(\sigma):=\lim _{\zeta \rightarrow 0} \frac{\xi(\zeta, \sigma) \cdot \zeta}{\zeta \cdot \sigma} \frac{\xi(\zeta, \sigma) \cdot \sigma}{|\sigma|} \\
& \quad=\left.\frac{\xi(0, \sigma) \cdot \sigma}{|\sigma|} \lim _{\zeta \rightarrow 0}\left(\frac{\zeta \cdot \xi}{\zeta \cdot \xi \alpha(\zeta, \xi)+|\zeta|^{2} \beta(\zeta, \xi)}\right)\right|_{\xi=\xi(\zeta, \sigma)}
\end{aligned}
$$

where

$$
\begin{aligned}
& \alpha(\zeta, \xi)=\int_{-1 / 2}^{1 / 2} \frac{P^{\prime}(|\xi+s \zeta|)}{|\xi+s \zeta|} d s \\
& \beta(\zeta, \xi)=\int_{-1 / 2}^{1 / 2} s \frac{P^{\prime}(|\xi+s \zeta|)}{|\xi+s \zeta|} d s
\end{aligned}
$$


Since $\beta(\zeta, \xi)=O(|\xi \cdot \zeta|)$, we conclude

$$
\begin{aligned}
L(\sigma) & =\frac{\xi(0, \sigma) \cdot \sigma}{|\sigma|} \frac{1}{\alpha(0, \xi(0, \sigma))} \\
& =\frac{\xi(0, \sigma) \cdot \sigma}{|\sigma|} \frac{|\xi(0, \sigma)|}{P^{\prime}(|\xi(0, \sigma)|)}
\end{aligned}
$$

Taking the limit $\tau_{1} \rightarrow-\infty, \tau_{2} \rightarrow+\infty$ in the righthand side of (3.4) now gives

$$
\begin{aligned}
& 2\left\langle\frac{\xi(0, \sigma) \cdot \sigma}{|\sigma|} \frac{|\xi(0, \sigma)|}{P^{\prime}(|\xi(0, \sigma)|)} D(0, \sigma)^{-1}, \hat{w}_{I}(0, \xi(0, \sigma))\right\rangle_{\sigma} \\
& \quad=2\left\langle\frac{|\xi|^{2}}{\left|P^{\prime}(|\xi|)\right|}, \hat{w}_{I}(0, \xi)\right\rangle_{\xi} \\
& \quad=\frac{2}{(2 \pi)^{n}} \int_{\mathbb{R}^{n}} \frac{|\xi|^{2}}{\left|P^{\prime}(|\xi|)\right|}\left|\hat{u}_{I}(\xi)\right|^{2} d \xi
\end{aligned}
$$

since $\sigma=\left(P^{\prime}(|\xi|) /|\xi|\right) \xi$ for $\zeta=0$.

It is an easy exercise to show that the condition of Theorem 3.1 are satisfied for $P(r)=r^{\nu}$ with $\nu \geq 2$ ( $c f$. [3], where also various other dispersion results for 1-dim-problems can be found). More generally we obtain

Corollary 3.1 Let $P \in C^{2}([0, \infty) ; \mathbb{R}),\left(\left(P^{\prime}(r) /\right.\right.$ $r)^{\prime} r \in C([0, \infty) ; \mathbb{R})$ and $P^{\prime}(r) \geq r^{2}\left|\left(P^{\prime}(r) / r\right)^{\prime}\right|$ for $r \geq 0$. Then (3.19) (a), (b) hold for the solution of (3.18) (a), (b) when the "=" sign in (3.2a) is replaced by " $\leq$ ".

Proof We set $Q(r)=\left(P^{\prime}(r) / r\right)$ and compute

$$
\begin{aligned}
\frac{\partial \sigma}{\partial \xi}(\zeta, \xi)= & \int_{-1 / 2}^{1 / 2} Q(|\xi+s \zeta|) d s I d \\
& +\int_{-1 / 2}^{1 / 2} \frac{Q^{\prime}(|\xi+s \zeta|)}{|\xi+s \zeta|}(\xi+s \zeta) \otimes(\xi+s \zeta) d s
\end{aligned}
$$

Therefore $(\partial \sigma / \partial \xi) \geq 0$ if $Q(r) \geq r\left|Q^{\prime}(r)\right|$ for $r>0$. If we replace $P(r)$ by $P^{\delta}(r)=P(r)+(1 / 2) \delta r^{2}$ for $\delta>0$ we have $\left(\partial \sigma^{\delta} / \partial \xi\right) \geq \delta I d$ everywhere. By the global implicit function Theorem the $\operatorname{map} \xi \rightarrow \sigma^{\delta}(\zeta, \xi)$ is a homeomorphism from $\mathbb{R}_{\xi}^{n}$ to $\mathbb{R}_{\sigma}^{n}$. Then the assertion follows by performing the limit $\delta \rightarrow 0+$.
Clearly the same result holds if $P^{\prime}(r) \leq-r^{2}$ $\left|\left(P^{\prime}(r) / r\right)^{\prime}\right|$ for all $r>0$.

Fourier-multipliers of the form $P(r)=r^{\nu}$ for $1 \leq \nu<2$ can be included in the theory be defining approximate symbols $P^{\delta}(r)$ as follows:

$$
\frac{d}{d r} P^{\delta}(r)=\nu \frac{r^{\nu}}{r+\delta} .
$$

A simple calculation shows that $P^{\delta}$ satisfies the assumptions of Corollary 3.1. The limit process $\delta \rightarrow 0$ then proves that the assertions of the Corollary also hold for $P\left(=\lim _{\delta \rightarrow 0} P^{\delta}\right)=r^{\nu}$.

A particularly interesting case is provided by $\nu=1$. We have for all $x_{0} \in \mathbb{R}^{n}(n \geq 2)$ :

$$
\begin{aligned}
\int_{-\infty}^{\infty}\left[\int_{\mathbb{R}^{n}}\right. & {\left[\frac{|\nabla u|^{2}}{\left|x-x_{0}\right|}-\frac{\left|\left(x-x_{0}\right) \cdot \nabla u\right|^{2}}{\left|x-x_{0}\right|^{3}}\right] d x } \\
+ & \left.\left\{\begin{array}{cc}
0, & n=2 \\
4 \pi\left|u\left(x_{0}, t\right)\right|^{2}, & n=3 \\
\alpha_{n} \int_{\mathbb{R}^{n}} \frac{|u|^{2}}{\left|x-x_{0}\right|^{3}} d x, & n \geq 4
\end{array}\right\}\right] d t \leq 2\left|u_{I}\right|_{1}^{2},
\end{aligned}
$$

and consequently

$$
\begin{aligned}
& \int_{-\infty}^{\infty} \int_{\mathbb{R}^{n}}\left[\frac{\left|\frac{\nabla}{|\nabla|} u\right|^{2}}{\left|x-x_{0}\right|}-\frac{\left|\left(x-x_{0}\right) \cdot \frac{\nabla}{|\nabla|} u\right|^{2}}{\left|x-x_{0}\right|^{3}}\right] d x d t \\
& \quad \leq 2\left|u_{I}\right|_{0}^{2}
\end{aligned}
$$

where $u$ is the solution of

$$
\begin{gathered}
i u_{t}=|D| u, \quad x \in \mathbb{R}^{n}, \quad t \in \mathbb{R} \\
u(t=0)=u_{I} .
\end{gathered}
$$

Remark 3.1 (Dirac equation) We consider the free Dirac equation in $\mathbb{R}^{3}$ for the spinorfield $U=U(x, t) \in \mathbb{C}^{4}$ :

$$
\begin{array}{r}
i U_{t}=R(D) U, \quad x \in \mathbb{R}^{3}, \quad t \in \mathbb{R} \\
U(t=0)=U_{I} \quad \text { on } \mathbb{R}^{3}
\end{array}
$$


where

$$
R(\xi)=\sum_{k=1}^{3} \gamma^{0} \gamma^{k} \xi_{k}+\gamma^{0}, \quad \xi=\left(\begin{array}{l}
\xi_{1} \\
\xi_{2} \\
\xi_{3}
\end{array}\right)
$$

$\gamma^{l}, l=0, \ldots, 4$, are the $4 \times 4$ Dirac matrices. Their elements are $0,1, i$ and they satisfy ( $c f$. [11]):

$$
\begin{aligned}
& \left(\gamma^{0}\right)^{*}=\gamma^{0}, \quad\left(\gamma^{k}\right)^{*}=-\gamma^{k} \\
& \left(\gamma^{0} \gamma^{k}\right)^{*}=\gamma^{0} \gamma^{k} \quad \text { for } k=1,2,3 \\
& \left(\gamma^{0}\right)^{2}=I d, \quad\left(\gamma^{k}\right)^{2}=-I d \quad \text { for } k=1,2,3 \\
& \gamma^{\mu} \gamma^{\nu}+\gamma^{\nu} \gamma^{\mu}=0 \quad \text { for } \mu \neq \nu
\end{aligned}
$$

Clearly $R(\xi)$ is self-adjoint for all $\xi \in \mathbb{R}^{3}$. Its eigenvalues are $\lambda_{+}(|\xi|)$ and $\lambda_{-}(|\xi|)$, where

$$
\lambda_{ \pm}(r)= \pm \sqrt{r^{2}+1}
$$

( $c f$. [9]). Each eigenvalue has multiplicity 2 for all $\xi \in \mathbb{R}^{3}$. Let $S_{+}(\xi), S_{-}(\xi)$ be the spectral projections of $R(\xi)$ corresponding to $\lambda_{+}(|\xi|)$ and $\lambda_{-}(\mid \xi)$ resp. Then we have

$$
U=U_{+}+U_{-}, \quad U_{+}=S_{+}(D) U, \quad U_{-}=S_{-}(D) U
$$

with the equations

$$
\begin{array}{ll}
i \frac{\partial}{\partial t} U_{+}=\lambda_{+}(|D|) U_{+}, & U_{+}(t=0)=S_{+}(D) U_{I} \\
i \frac{\partial}{\partial t} U_{-}=\lambda_{-}(|D|) U_{-}, & U_{-}(t=0)=S_{-}(D) U_{I} .
\end{array}
$$

Since $\lambda_{+}(r),-\lambda_{-}(r)$ satisfy the assumption of Corollary 2.1, we obtain the estimate (3.2a) (for $n=3$ with the equal sign replaced by " $\leq$ ") for all 4 components of $U_{+}$and $U_{-}$. Proceeding as for the wave equation gives for all $x_{0} \in \mathbb{R}^{3}$ :

$$
\int_{-\infty}^{\infty}\left|U\left(x_{0}, t\right)\right|^{2} d t \leq \frac{1}{4 \pi}\left|U_{I}\right|_{1}^{2}
$$

which proves the 'regularizing' effect of the Dirac equation from $H^{1}\left(\mathbb{R}^{3}\right)$ (for the initial data) to $L^{\infty}\left(\mathbb{R}_{x}^{3} ; L^{2}\left(\mathbb{R}_{t}\right)\right)$. Also the term involving the singularity at $x=x_{0}$ in (3.2a) can be computed. We have

$$
S_{ \pm}(\xi)=\frac{1}{2}\left(I \pm \frac{1}{\sqrt{|\xi|^{2}+1}} R(\xi)\right)
$$

and thus:

$$
\begin{aligned}
& \int_{-\infty}^{\infty} \int_{\mathbb{R}^{3}}\left(\frac{\left|\frac{\nabla}{|\nabla|} U_{ \pm, l}\right|^{2}}{\left|x-x_{0}\right|}-\frac{\left|\left(x-x_{0}\right) \cdot \frac{\nabla}{|\nabla|} U_{ \pm, l}\right|^{2}}{\left|x-x_{0}\right|^{3}}\right) d x d t \\
& \quad \leq \text { const }\left|U_{I}\right|_{0}^{2}
\end{aligned}
$$

for $l=1,2,3,4$, where $U_{ \pm}=\left(U_{ \pm, 1}, U_{ \pm, 2}, U_{ \pm, 2}\right.$, $\left.U_{ \pm, 3}\right)^{T}$.

\section{Acknowledgement}

This research was supported by the German DAAD program PROCOPE titled Verallgemeinerte Halbleitermodelle. The first and the second author were also supported by grant $\mathrm{Nr}$. MA 1662/1-2 and 2-2 of the 'Deutsche Forschungsgemeinschaft' and by grant Nr. 315/PPP/ru-ab of the German 'DAAD'. The third author was partially supported by TMR project $\mathrm{HCL} \mathrm{Nr}$. ERBFMRXCT960033.

\section{References}

[1] Ben-Arzi, M. and Klainerman, S. (1997). Regularity and decay of evolution equations, to appear in J. Anal. Math.

[2] Ben Arzi, M. and Devinatz, A. (1991). Local Smoothing and Convergence Properties of Schrödinger Type Equations, J. Funct. Anal., 101, 231-245.

[3] Colin, T. (1994). Smoothing effects for dispersive equations via a generalized Wigner transform, SIAM J. Math. Anal., 25, 1622-1641.

[4] Constantin, P. and Saut, J. C. (1988). Local smoothing properties of dispersive equations, J. Amer. Math. Soc., 1, 413-446.

[5] Constantin, P. and Saut, J. C. (1989). Local smoothing properties of Schrödinger equations, Indiana Univ. Math. Journal, 38, $791-810$.

[6] Cazenave, T. (1993). An introduction to nonlinear Schrödinger Equations, Second Edition, Textos de Métodos Matemàticas 26, Universidad Federal do Rio de Janeiro.

[7] Gasser, I., Illner, R., Markowich, P. A. and Schmeiser, C. (1996). Semiclassical, $t \rightarrow \infty$ asymptotics and dispersive effects for Hartree-Fock Systems, M $^{2}$ AN, 32, 699-713. 
[8] Gasser, I. and Markowich, P. A. (1997). Quantum Hydrodynamics, Wigner Transforms and the Classical Limit, Asymptotic Analysis, 14, 97-116.

[9] Gérard, P., Markowich, P. A., Mauser, N. J. and Poupaud, F. (1997). Homogenization limits and Wigner transforms, Comm. Pure Appl. Math., 50, 321-357.

[10] Gasser, I., Markowich, P. A. and Perthame, B. (1997). Dispersion and moment lemmas revisited, submitted.

[11] Landau, L. D. and Lifschitz, E. M., Lehrbuch der Theoretischen Physik IVa (Akademie-Verlag 1985), 7th edition.

[12] Lions, P. L. et Paul, T. (1993). Sur les mesures de Wigner, Revista Matematica Iberoamericana, 9, 553-618.

[13] Lions, P. L. et Perthame, B. (1992). Lemme de moments, de moyenne et de dispersion, C.R. Acad. Sci. Paris, 314, Série 1, 801-806.

[14] Lin, J. E. and Strauss, W. A. (1978). Decay and scattering of solutions of a nonlinear Schrödinger equation, J. Funct. Anal., 30, 245-263.

[15] Markowich, P. A. and Mauser, N. J. (1993). The classical limit of a self-consistent quantum-Vlasov equation in $3 \mathrm{~d}$, $M^{3}$ AS, 3, 109-124.

[16] Perthame, B. (1996). Time decay, propagation of low moments and dispersive effects of kinetic equations, Comm. PDE. 21, 659-686.

[17] Reed, M. and Simon, B. (1975). Methods of modern mathematical physics II, Fourier analysis, self-adjointness, Academic Press, New York.

[18] Ruiz, A. and Vega, L. (1994). Local regularity of solutions to wave equations with time-dependent potentials, Duke Math. Journal, 76.

[19] Stein, E. M. (1971). Singular integrals and differentiability properties of functions, Princeton Univ. Press, Princeton, N.J.

[20] Sjölin, P. (1987). Regularity of solutions to the Schrödinger equation, Duke Math. J., 55, 699-715.

[21] Sjögren, P. and Sjölin, P., Convergence properties for the time dependent Schrödinger Equation, to appear in Ann. Acad. Sci. Fenn.
[22] Vega, L. (1988). Schrödinger equations: Pointwise convergence to the initial data, Proc. Amer. Math. Soc., 102, $874-878$.

\section{Authors' Biographies}

I. Gasser received his Ph.D. in Mathematics from the Technical University of Berlin (Germany) in 1996. He is currently an Assistant Professor at the Institut für Angewandte Mathematik at the University of Hamburg (Germany). His fields of interest are partial differential equations, semiconductor models and kinetic theory.

P. A. Markowich received his Ph.D. in Applied Mathematics from the Technical University of Vienna (Austria) in 1980. He was Professor of Mathematics at the Technical University of Berlin (Germany) from 1989 to 1998 and is currently Professor of Mathematics at the University of Linz (Austria). His fields of interest are partial differential equations and kinetic theory.

B. Perthame completed his these d'etat at the University Paris Dauphine in 1987. He became Professor of Mathematics at the University d'Orleans in 1988. He is currently Professor of Mathematics at the Ecole Normale Superieure in Paris. His main fields of interest are partial differential equations, their applications and numerical analysis. 

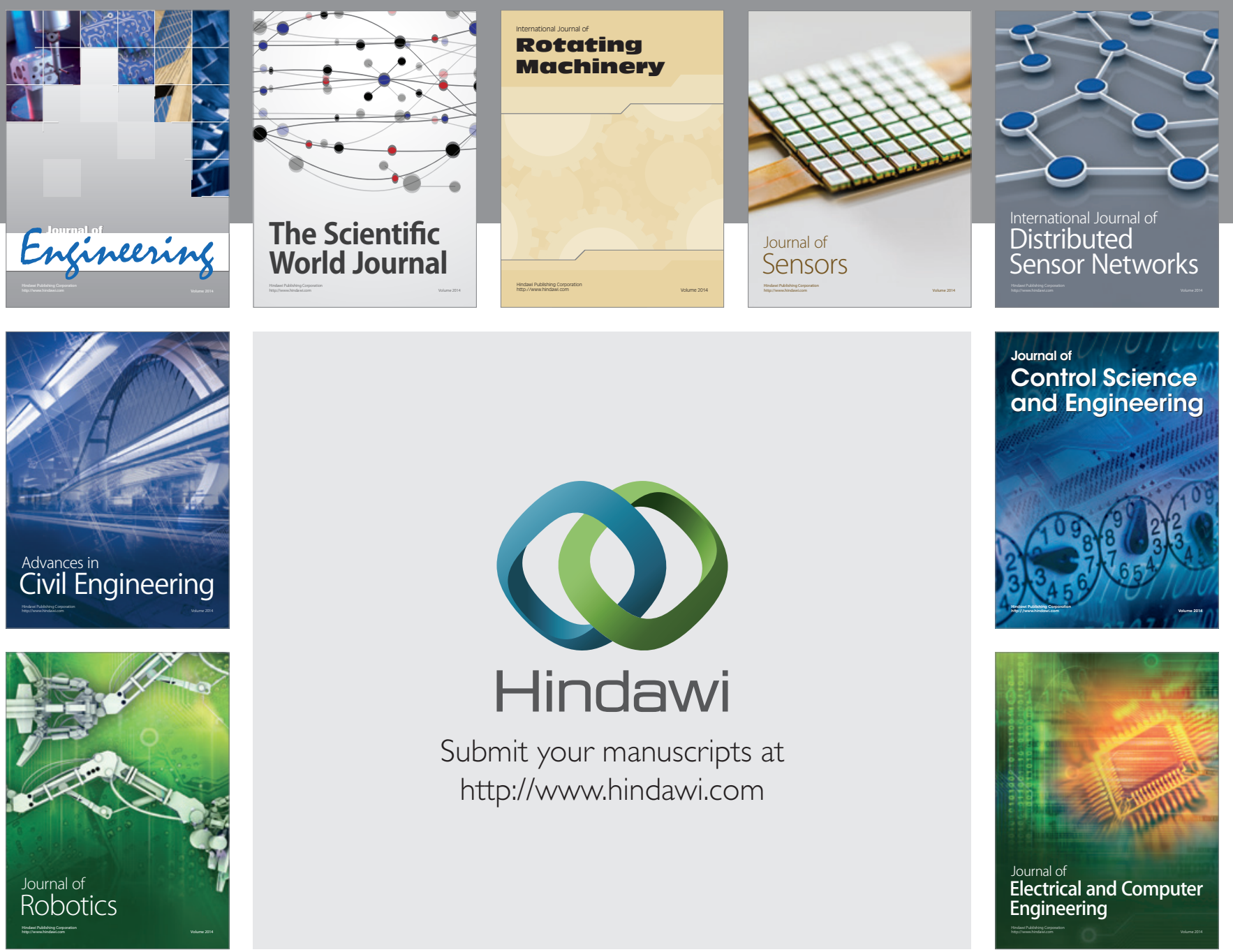

Submit your manuscripts at

http://www.hindawi.com
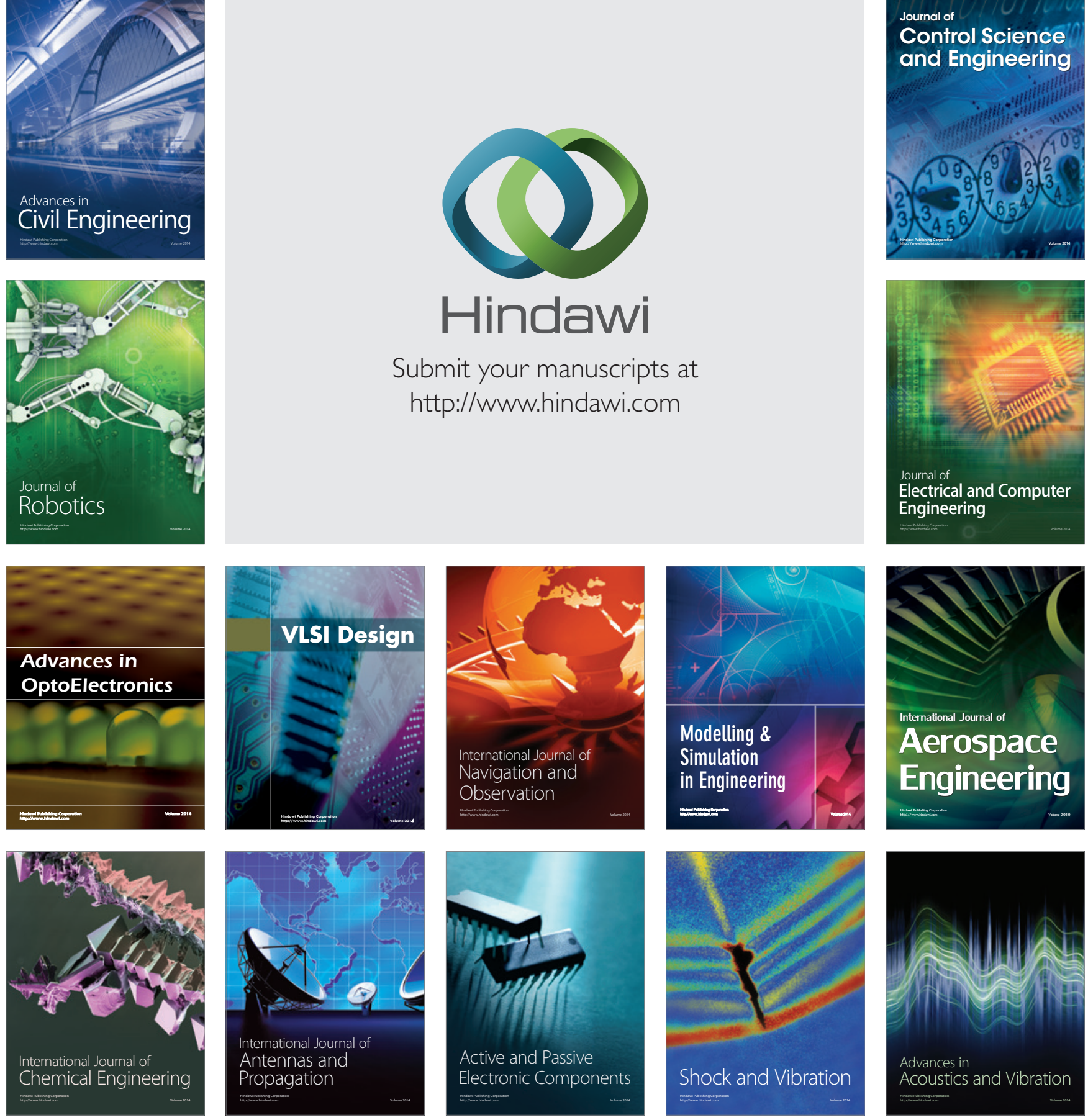